\title{
Waves at a fluid-solid interface: Explicit versus implicit formulation of boundary conditions using a discontinuous Galerkin method
}

Khemraj Shukla, ${ }^{1}$ José M. Carcione, ${ }^{2}$ Jan S. Hesthaven, ${ }^{3}$ and Elizabeth L'heureux ${ }^{4}$

${ }^{1}$ Center of Computation and Visualization, Brown University, 180 George St,

Providence, RI 02906

${ }^{2}$ Istituto Nazionale di Oceanografia e di Geofisica Sperimentale (OGS),

Borgo Grotta Gigante 42c, 34010 Sgonico, Trieste, Italy.

${ }^{3}$ Computational Mathematics and Simulation Sciences (MCSS), EPFL,

Switzerland

${ }^{4}$ Complex Imaging Group, BP America, Houston, TX 77079 
An accurate solution of the wave equation at a fluid-solid interface requires a correct implementation of the boundary condition. Boundary conditions at acousto-elastic interface require continuity of the normal component of particle velocity and traction, whereas the tangential components vanish. The main challenge is to model interface waves, namely the Scholte and leaky Rayleigh waves. In this study, we use a nodal discontinuous Galerkin (dG) finite-element method with the medium discretized using an unstructured uniform triangular meshes. The natural boundary conditions in the dG method is implemented by 1) using an explicit upwind numerical flux and 2) by using an implicit penalty flux and setting the modulus of rigidity of the acoustic medium to zero. The accuracy of these methods are evaluated by comparing the numerical solutions with analytical ones, with source and receiver at and away from the interface. The study shows that the solution obtained from the explicit and implicit boundary conditions produces the correct results. This is due to the fact that the stability of the $\mathrm{dG}$ scheme is determined the numerical flux, which also implements the boundary conditions by unifying the numerical solution at shared edges of the elements in an energy stable manner.

arajexplo@gmail.com 


\section{INTRODUCTION}

The accurate computation of wave fields in realistic Earth models is an ongoing challenge in local and global seismology. The problem becomes more challenging in the presence of fluid-solid interfaces. Of particular interest, are applications in geophysics i.e., marine seismic exploration concerning the ocean bottom or global Earth seismology, using earthquakegenerated seismic waves as a probing field, involving the core-mantle-boundary (CMB) and inner-core-boundary (ICB). In a fluid-solid setup, there exist two interface waves. First, the "Scholte waves" that mainly travels into the liquid (Cagniard, 1962), and, second is, the leaky-Rayleigh wave (Ash and Paige, 1985). The latter wave decays with depth in the solid while it behaves as a propagating mode in the fluid (Ash and Paige, 1985) with characteristics similar to the head waves. Surprisingly, the amplitude of the leaky-Rayleigh mode in fluid increases as it propagates away from the interface (Farnell, 1970).

The main physical aspects of the interface waves are discussed in (Glorieux et al., 2002, 2001; Padilla et al., 1999). In subsequent sections, the bulk velocities of the longitudinal waves in fluid and solid are represented by $c_{f}$ and $c_{P}$, respectively, the shear-wave velocity of the solid is represented by $c_{S}$, and the phase velocity of the leaky Rayleigh wave, free Rayleigh waves in the solid and Scholte wave are denoted by $c_{p R}, c_{R}$ and $c_{S c}$, respectively. The findings of (Glorieux et al., 2002, 2001; Padilla et al., 1999) are summarized as: 
1. In the case of $c_{f}<c_{S}<c_{p}$, the stiff solid condition, the leaky-Rayleigh wave propagates with a phase velocity $\left(c_{p R}\right)$ slightly lower than $c_{S}$ and leaks energy in the form of the head wave.

2. In the case of $c_{s}<c_{f}<c_{P}$, the soft solid condition, Padilla et al. (Padilla et al., 1999) state that the leaky Rayleigh mode travels in the fluid without dissipation as it is described by a real root. This unphysical observation is refuted by (Glorieux et al., 2001) with the argument that the root corresponding to the leaky Rayleigh mode is incorrectly identified.

3. If the density of the fluid $\rho_{f}$ is close to zero, the leaky Rayleigh wave becomes a free Rayleigh wave.

4. In the case of a large contrast in the acoustic impedance of the solid and fluid, the phase velocity of the Scholte wave $c_{S c}$ is close to $c_{f}$.

5. For the case of a soft solid $c_{S}<c_{f}<c_{p}$, the phase velocity of the Scholte wave is less than $c_{f}$ and the wave becomes more localized.

6. In the $2 \mathrm{D}$ case, the Scholte waves travel without attenuation whereas in 3D the dissipation of energy is caused by geometrical spreading.

In brief, for a stiff solid it holds that $c_{S c}<c_{R}<c_{p R}<c_{f}<c_{S}<c_{P}$. The domain of existence of the leaky-Rayleigh mode is described by the Rayleigh window defined by the angle between between the front of the Rayleigh wave and the interface (Carcione and Helle, 2004). 
A variety of numerical methods are used to solve the acousto-elastic system. The discretization is performed on (a) first-order system of partial differential equations (PDEs) in the stress-velocity formulation, or (b) in its original form of displacement, described by a system of second order PDEs. A detailed discussion on the numerical methods adopted for simulation of interface waves in a fluid-solid setup is discussed in a recent study by Carcione et al. (Carcione et al., 2018). The first to model the interface was Stephen (Stephen, 1983). He used a second-order finite-difference method to solve the elastic wave equation and treated the boundary condition by approximating the interface with a transition zone where the velocity increases gradually. Stephen also treated the boundary condition by setting $c_{S}=0$ in the acoustic media but obtained erroneous results. A spectral finite element method was used by Komatitsch et al., (Komatitsch et al., 2000) who treated the boundary conditions explicitly. Komatitsch et al. (Komatitsch et al., 2000) do not show the propagation of interface waves and lacks the comparison between numerical and analytical solutions. van Vossen et al. (van Vossen et al., 2002) used an explicit method based on finite differences, where only five grid points per wavelength are required for an accurate modeling. Zhang (Zhang, 2004) used an integral-based approach to model the boundary conditions explicitly with a domain discretized by unstructured meshes. The study presented by van Vossen et al. (Zhang, 2004) and Zhang (Zhang, 2004) do not show the propagation of interface waves (Scholte and leaky Rayleigh waves) and also do not show the accuracy of the numerical solutions by comparison to the analytical solution. Carbajal-Romero et al. (Carbajal-Romero et al., 2013) use a boundary element method to simulate the body and Scholte waves (ex- 
cluding the leaky Rayleigh wave) and validate their results by comparing the results against solutions obtained by using a discrete wave-number method and a spectral-element method. Chaljub et al. (Chaljub et al., 2003) solves the system for a heterogenous fluid-solid sphere but did not simulate the interface waves discussed here. Madec et al. (Madec et al., 2009) solve the system by using a high-order finite element method and primarily simulated the body waves but do not consider the interface waves. DeBasabe and Sen (De Basabe and Sen, 2014) has modeled the Scholte waves and compared the solutions obtained with several algorithms such as finite differences and spectral-element methods, which show limited accuracy. They concluded that the single-grid method have spurious modes in the fluid subdomain, but they do not show any comparison or results obtained by an explicit method.

Recently, Carcione et al. (Carcione et al., 2018) also simulated the interface waves using a direct-grid method, based on Chebyshev spatial differentiation, by incorporating natural boundary conditions using implicit and explicit approaches. In the implicit method, the natural boundary condition is incorporated by setting $c_{S}=0$ in the acoustic medium (fluid). The explicit method is based on a domain decomposition technique (Carcione, 2014). They concluded that the implicit method gives erroneous numerical results by comparing the numerical and analytical solutions, whereas the explicit method worked well.

Unlike the methods discussed above, which recover the numerical solution globally, we consider the discontinuous Galerkin $(\mathrm{dG})$ method. In the $\mathrm{dG}$ method, the global solution is obtained by unifying the solution at the shared edges of the elements by using a weak operator, known as a numerical flux (Hesthaven and Warburton, 2007, page 34). The 
boundary and interface conditions are incorporated in the scheme through this operator. The numerical flux determines the stability of the numerical scheme and thus ensures a correct implementations of the interface boundary conditions. For a linear problem, the flux can be computed explicitly by solving a Riemann problem on the interior boundary of each element (Wilcox et al., 2010), discretizing the acousto-elastic domain, resulting in an upwind flux. The computation of the upwind flux is expensive as it involves the eigen-decomposition, which becomes more cumbersome for anisotropic media and the extension is non-trivial. To circumvent this problem, Ye et al. (Ye et al., 2016) proposed an explicit method to compute the numerical flux by adding a penalty term to the central flux (jump in the value of the field variables in the direction normal to the edge of the element), which eventually stabilizes the numerical scheme. In this study, we compute the numerical solutions using both fluxes, upwind and penalized central fluxes, and show that in both cases the $\mathrm{dG}$ method captures the interface waves accurately.

The paper is organized as follows. Section II outlines the acousto-elastic system and Section III reviews the dispersion equation for interface waves. Section IV describes the system of equations in matrix form. Section $V$ formulates the $d G$ scheme for a coupled acoustic-elastic system, and Section VI presents the results. In VII, we offer a few concluding remarks. 


\section{SYSTEM OF EQUATIONS DESCRIBING ACOUSTIC-ELASTIC WAVES}

The two-dimensional velocity-stress equations for elastic wave propagation in the $(x, z)$ plane can be expressed by:

i) Euler-Newton equations:

$$
\begin{aligned}
& \dot{v}_{1}=\frac{1}{\rho}\left(\frac{\partial \sigma_{x x}}{\partial x_{1}}+\frac{\partial \sigma_{x z}}{\partial x_{3}}\right), \\
& \dot{v}_{2}=\frac{1}{\rho}\left(\frac{\partial \sigma_{x z}}{\partial x_{1}}+\frac{\partial \sigma_{z z}}{\partial x_{3}}\right),
\end{aligned}
$$

where $v_{1}$ and $v_{2}$ are the particle velocities along the $x$ and $z$ axis, $\sigma_{x x}, \sigma_{z z}$ and $\sigma_{x z}$ are stress components and $\rho$ is the density. A dot above a variable denotes the time derivative.

ii) Constitutive equations:

$$
\begin{aligned}
& \dot{\sigma}_{x x}=(\lambda+\mu)\left(\frac{\partial v_{1}}{\partial x_{1}}+\frac{\partial v_{2}}{\partial x_{3}}\right)+\mu\left(\frac{\partial v_{1}}{\partial x_{1}}-\frac{\partial v_{2}}{\partial x_{3}}\right)+f_{x x} \\
& \dot{\sigma}_{z z}=(\lambda+\mu)\left(\frac{\partial v_{1}}{\partial x_{1}}+\frac{\partial v_{2}}{\partial x_{3}}\right)-\mu\left(\frac{\partial v_{1}}{\partial x_{1}}-\frac{\partial v_{2}}{\partial x_{3}}\right)+f_{x z} \\
& \dot{\sigma}_{x z}=\mu\left(\frac{\partial v_{1}}{\partial x_{3}}+\frac{\partial v_{2}}{\partial x_{1}}\right)+f_{z z}
\end{aligned}
$$

where $\lambda$ and $\mu$ are the Lame's constants, and $f_{i j}$ are moment forces. In $2 \mathrm{D} \lambda=\rho\left(C_{L}^{2}-2 C_{S}^{2}\right)$ and $\mu=\rho C_{S}^{2}$, where $C_{L}$ and $C_{S}$ are the compressional and shear wave velocities, respectively. 


\section{THE DISPERSION EQUATION}

The dispersion equation is obtained by implementing the continuity of the normal components of the displacement and stress at the interface. This is expressed as (Carcione et al., 2018; Carcione and Helle, 2004; Glorieux et al., 2002, 2001; Scholte, 1942)

$$
S(q)=4 \sqrt{(1-q)(1-a q)}-(2-q)^{2}-\left(\frac{\rho_{f}}{\rho}\right) q^{2} \sqrt{\frac{1-a q}{1-b q}}
$$

where $q=\frac{v^{2}}{c_{S}^{2}}, a=\frac{c_{S}^{2}}{c_{L}^{2}}$ and $b=\frac{c_{S}^{2}}{c_{f}^{2}}$ with $v$ being the complex velocity of the interface waves.

The dispersion equation of the elastic Rayleigh wave is obtained by taking $\rho_{f}=0$ in (6). The Riemann surface of $S(q)$ has eight sheets, corresponding to different choices of the signs of the complex roots $\sqrt{1-q}, \sqrt{1-a q}$ and $\sqrt{1-b q}$ (Ansell, 1972). In the lossless case, the roots of the Scholte and leaky Rayleigh waves are real and complex, respectively. These surface waves are a superposition of inhomogeneous elastic waves and the attenuation vector makes an angle of $90^{\circ}$ with respect to the propagation vector. The Scholte wave attenuates in the direction perpendicular to the interface, while the leaky Rayleigh wave attenuates along the interface. The Scholte waves travels without attenuation in 2D lossless media, whereas in 3D media the energy of Scholte waves decays due to geometrical spreading. The energy of the leaky Rayleigh wave decays both in 2D and 3D losless media.

The existence of the leaky wave is subject to the condition that the sound velocity in the liquid must be less than the shear velocity in the solid. When the density of the liquid tends to zero, the leaky Rayleigh approaches the Rayleigh wave. Thus, for a stiff bottom (shear velocity in the solid higher than the velocity in water), the Rayleigh wave is not the Scholte 


\section{SYMMETRIC FORM OF THE SYSTEM OF EQUATION IN MATRIX FORM}

Equations (1)-(5) can be rewritten as a symmetric hyperbolic system of PDEs using the Voigt notation:

$$
\begin{gathered}
\rho \frac{\partial \boldsymbol{v}}{\partial t}=\sum_{i=1}^{2} \boldsymbol{A}_{i}^{T} \frac{\partial \boldsymbol{\sigma}}{\partial x_{i}} \\
\boldsymbol{C}^{-1} \frac{\partial \boldsymbol{\sigma}}{\partial t}=\sum_{i=1}^{2} \boldsymbol{A}_{i} \frac{\partial \boldsymbol{v}}{\partial \boldsymbol{x}_{i}}+\boldsymbol{f}
\end{gathered}
$$




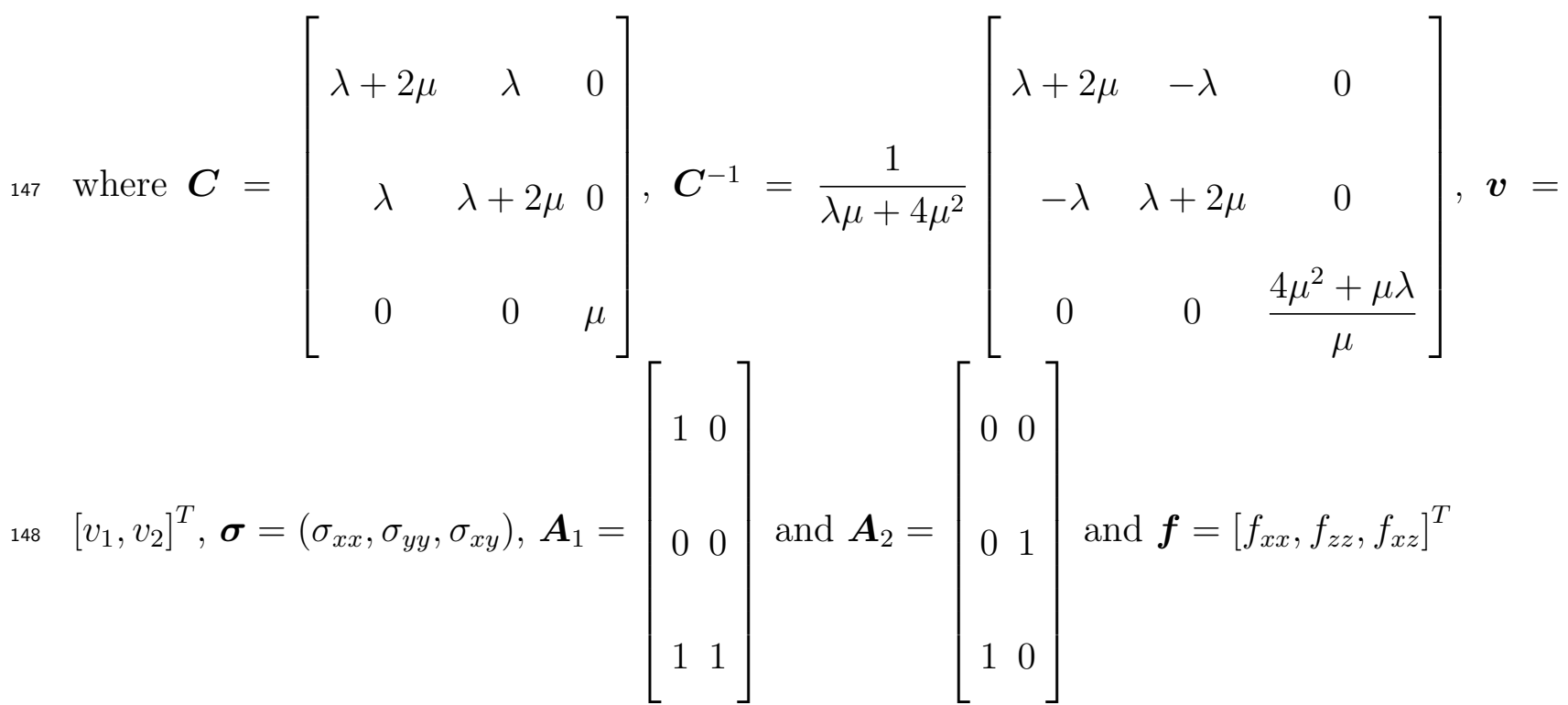
149 Note that $\boldsymbol{C}$ is symmetric and positive-definite. Matrices $\boldsymbol{A}_{1}$ and $\boldsymbol{A}_{2}$ are constant but ${ }_{150} \rho, \boldsymbol{C}$ and $\boldsymbol{C}^{-1}$ may vary spatially.

Combining equations (7) and (8) results

$$
\boldsymbol{Q} \frac{\partial \boldsymbol{q}}{\partial t}+\nabla \cdot(\boldsymbol{A q})=\boldsymbol{g}
$$

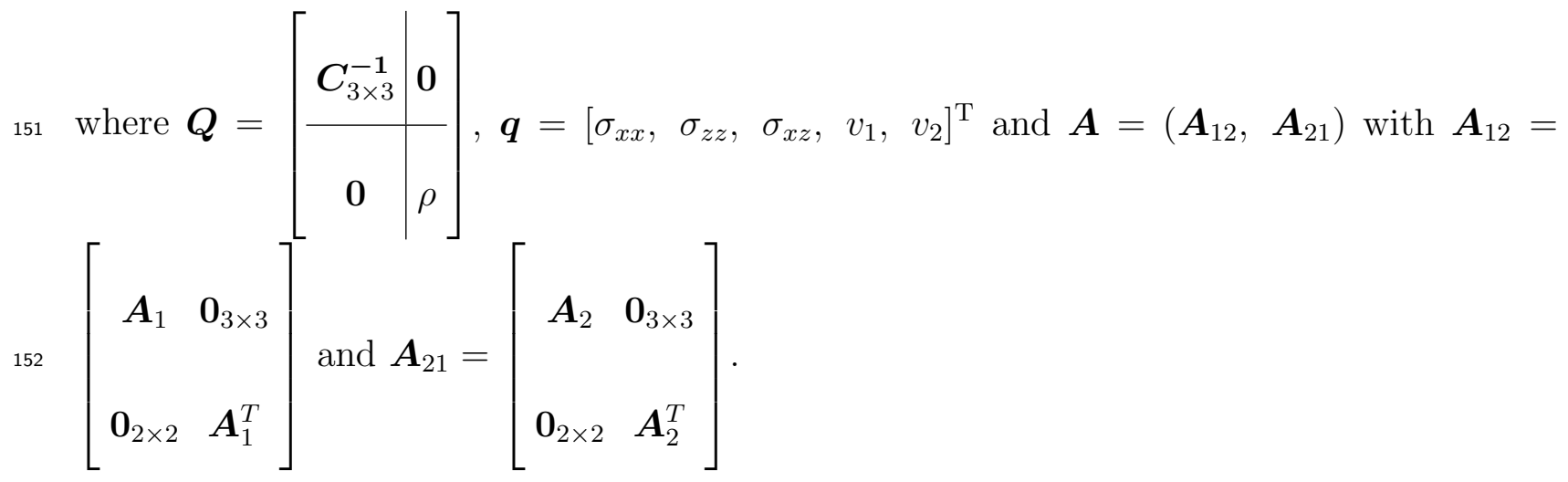




\section{A DISCONTINUOUS GALERKIN FORMULATION FOR ACOUSTO-ELASTIC} WAVE PROPAGATION

We assume that the domain $\Omega$ is triangulated by a mesh $\Omega_{h}$ which consists of elements $D^{k}$ which are images of a reference element $\hat{D}$ under the local affine mapping.

$$
\boldsymbol{x}^{k}=\Phi^{k} \widehat{\boldsymbol{x}}
$$

where $\boldsymbol{x}^{k}=\left\{x^{k}, y^{k}\right\}$ for $d=2$ denote the physical coordinates on $D^{k}$ and $\hat{\boldsymbol{x}}=\{\hat{x}, \hat{y}\}$ for $d=2$ and $\widehat{\boldsymbol{x}}=\{\widehat{x}, \widehat{y}, \widehat{z}\}$ for $d=3$ denote coordinates on the reference element. We denote the determinant of the Jacobian of $\Phi^{k}$ as $J$.

Solutions over each element $D^{k}$ are approximated from a local approximation space $V_{h}\left(D^{k}\right)$, which is defined as a composition of the mapping $\Phi^{k}$ and a reference approximation space $V_{h}(\widehat{D})$

$$
V_{h}\left(D^{k}\right)=\Phi^{k} \circ V_{h}(\widehat{D})
$$

The global approximation space $V_{h}\left(\Omega_{h}\right)$ is defined as

$$
V_{h}\left(\Omega_{h}\right)=\bigoplus_{k} V_{h}\left(D^{k}\right)
$$

We take $V_{h}(\widehat{D})=P^{N}(\widehat{D})$, with $P^{N}(\widehat{D})$ being the space of polynomials of total degree $N$ on the reference simplex. In two dimensions, $P^{N}$ on a triangle is

$$
P^{N}(\widehat{D})=\left\{\widehat{x}^{i} \widehat{y}^{j}, 0 \leq i+j \leq N\right\}
$$

The $L^{2}$ inner product and norm over $D^{k}$ is represented as

$$
(\boldsymbol{g}, \boldsymbol{h})=\int_{D^{k}} \boldsymbol{g} \cdot \boldsymbol{h} d \boldsymbol{x}=\int_{\hat{D}} \boldsymbol{g} \cdot \boldsymbol{h} J d \hat{\boldsymbol{x}}, \quad\|\boldsymbol{g}\|_{L^{2} \Omega}^{2}=(\boldsymbol{g}, \boldsymbol{g})_{L^{2}\left(D^{k}\right)}
$$


where $\boldsymbol{g}$ and $\boldsymbol{h}$ are vector-valued real functions. Global $L^{2}$ products and squared norms are defined as the sum of local $L^{2}$ inner products and squared norms over each element. The $L^{2}$ inner product and norm over the boundary $\partial D^{k}$ of an element are similarly defined as

$$
\langle\boldsymbol{u}, \boldsymbol{v}\rangle_{L^{2}\left(\partial D^{k}\right)}=\int_{\partial D^{k}} \boldsymbol{u} \cdot \boldsymbol{v} d \boldsymbol{x}=\sum_{f \in \partial D^{k}} \int_{\hat{f}} \boldsymbol{u} \cdot \boldsymbol{v} J^{f} d \hat{\boldsymbol{x}}, \quad\|\boldsymbol{u}\|_{L^{2}\left(\partial D^{k}\right)}^{2}=\langle\boldsymbol{u}, \boldsymbol{u}\rangle
$$

where $J^{f}$ is the Jacobian of the mapping from a reference face $\hat{f}$ to a physical face $f$ of an element.

Let $f$ be a face of element $D^{k}$ with neighboring element $D^{k,+}$ and unit outward normal $\boldsymbol{n}$. Let $u$ be a piecewise smooth function with discontinuities across element interfaces. We define the interior value $u^{-}$and exterior value $u^{+}$on face $f$ of $D^{k}$,

$$
u^{-}=\left.u\right|_{f \cap \partial D^{k}}, \quad u^{+}=\left.u\right|_{f \cap \partial D^{k,+}}
$$

The jump and average of a scalar function $u \in V_{h}\left(\Omega_{h}\right)$ over $f$ are then defined as

$$
\llbracket u \rrbracket=u^{+}-u^{-}, \quad\{\{u\}\}=\frac{u^{+}+u^{-}}{2}
$$

respectively. Jumps and averages of vector-valued functions $\boldsymbol{u} \in \mathbb{R}^{m}$ and matrix-valued functions $\tilde{\boldsymbol{S}} \in \mathbb{R}^{m \times n}$ are defined component-wise,

$$
(\llbracket \boldsymbol{u} \rrbracket)_{i}=\llbracket \boldsymbol{u}_{i} \rrbracket, \quad 1 \leq i \leq m \quad(\llbracket \tilde{\boldsymbol{S}} \rrbracket)_{i j}=\llbracket \tilde{\boldsymbol{S}} \rrbracket
$$


The strong dG formulation on an element $D^{k}$ is obtained by integration by parts of (9) twice, in the space on each element $D^{k}$,

$$
\int_{D^{k}} \boldsymbol{Q} \frac{\partial \boldsymbol{q}}{\partial t} \cdot \boldsymbol{p} d \boldsymbol{x}+\int_{D^{k}}(\nabla \cdot \boldsymbol{A q}) \cdot \boldsymbol{p} d x+\int_{\partial D^{k}}\left(\boldsymbol{n} \cdot\left((\boldsymbol{A q})^{*}-\boldsymbol{A}^{-} \boldsymbol{q}^{-}\right)\right) \cdot \boldsymbol{p} d \boldsymbol{x}=\int_{D^{k}} \boldsymbol{f} \cdot \boldsymbol{p} d \boldsymbol{x}
$$

for all $\boldsymbol{p} \in \boldsymbol{V}_{h}\left(\Omega_{h}\right)$,

\section{A. Explicit scheme}

To compute the upwind flux, we solve a Riemann problem at shared edges of each element, which corresponds to an elastic-elastic, acoustic-acoustic, acoustic-elastic and elastic-acoustic interface. The Riemann problem to solve (10) is defined as

$$
\boldsymbol{q}_{0}(\boldsymbol{x})=\left\{\begin{array}{l}
\boldsymbol{q}^{-} \text {if } \boldsymbol{n} \cdot \boldsymbol{x}<0 \\
\boldsymbol{q}^{+} \text {if } \boldsymbol{n} \cdot \boldsymbol{x}>0
\end{array}\right.
$$

The solution of (11) is described separately for elastic-elastic, acoustic-acoustic and elasticacoustic or acoustic elastic-interfaces

\section{Elastic-elastic interfaces}

Equation (9) is written as

$$
\boldsymbol{Q} \frac{\partial \boldsymbol{q}}{\partial t}+\frac{\partial \boldsymbol{A}_{12}}{\partial x_{1}}+\frac{\partial \boldsymbol{A}_{21}}{\partial x_{2}}=\boldsymbol{f}
$$




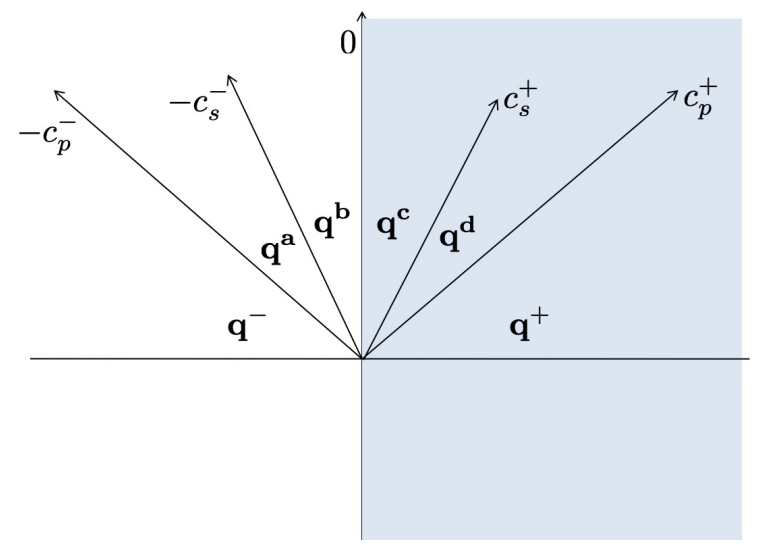

FIG. 1. Rankine-Hugonite jump conditions in the Riemann's problem

In the remainder of this section, $\boldsymbol{n}:=\boldsymbol{n}^{-}$denotes the outward interface unit normal vector and the flux in the normal direction is then $\boldsymbol{A}_{n} \boldsymbol{q}$ with $\boldsymbol{A}_{n}=n_{1} \boldsymbol{A}_{12}+n_{2} \boldsymbol{A}_{21}$. To construct an upwind flux requires the computations of the characteristics of the hyperbolic system defined in (9). To compute the characteristics, we need the eigen-decomposition of $\boldsymbol{Q}^{-1} \boldsymbol{A}_{n}$ expressed as

$$
\boldsymbol{Q}^{-1} \boldsymbol{A}=\boldsymbol{R} \Lambda \boldsymbol{R}^{-1}
$$

with $\Lambda=\operatorname{diag}\left(-c_{P},-c_{S}, 0, c_{S}, c_{P}\right)$ and the $j^{\text {th }}$ column of $\boldsymbol{R}$ is an eigenvector of $\boldsymbol{Q}^{-1} \boldsymbol{A}$. For each wave speed $c_{i}$ in our system, the Rankine-Hugonoit jump condition is expressed as

$$
-c_{i} \boldsymbol{Q}\left(\boldsymbol{q}^{m}-\boldsymbol{q}^{p}\right)+\boldsymbol{A}_{n}\left(\boldsymbol{q}^{m}-\boldsymbol{q}^{p}\right)=0
$$

where, $\boldsymbol{q}^{m}$ and $\boldsymbol{q}^{p}$ are states in the negative and positive normal directions, travelling with speed $c_{i}$, across the discontinuity. Matrices $\boldsymbol{Q}$ and $\boldsymbol{A}_{n}$ are evaluated in the region where mode $c_{i}$ travels, denoted by "-" and "+". The Riemann problem has four unknown states 
$\left(\boldsymbol{q}^{a}, \boldsymbol{q}^{b}, \boldsymbol{q}^{c}, \boldsymbol{q}^{d}\right)$ as described in Figure 1. A full set of Rakine-Hugonite jump conditions is given as

$$
\begin{array}{r}
c_{P}^{-} \boldsymbol{Q}^{-}\left(\boldsymbol{q}^{-}-\boldsymbol{q}^{a}\right)+\boldsymbol{A}_{n}^{-}\left(\boldsymbol{q}^{-}-\boldsymbol{q}^{a}\right)=0 \\
c_{S}^{-} \boldsymbol{Q}^{-}\left(\boldsymbol{q}^{a}-\boldsymbol{q}^{b}\right)+\boldsymbol{A}_{n}^{-}\left(\boldsymbol{q}^{a}-\boldsymbol{q}^{b}\right)=0 \\
\boldsymbol{A}_{n}^{-} \boldsymbol{q}^{b}-\boldsymbol{A}_{n}^{+} \boldsymbol{q}^{c}=0 \\
-c_{S}^{+} \boldsymbol{Q}^{+}\left(\boldsymbol{q}^{a}-\boldsymbol{q}^{b}\right)+\boldsymbol{A}_{n}^{+}\left(\boldsymbol{q}^{c}-\boldsymbol{q}^{d}\right)=0 \\
-c_{P}^{+} \boldsymbol{Q}^{+}\left(\boldsymbol{q}^{d}-\boldsymbol{q}^{+}\right)+\boldsymbol{A}_{n}^{+}\left(\boldsymbol{q}^{d}-\boldsymbol{q}^{+}\right)=0
\end{array}
$$

Using the definitions of the eigenvalues and eigenvectors of $\left(Q^{-}\right)^{-1} A^{-}$and $\left(Q^{+}\right)^{-1} A^{+}$, we obtain

$$
\begin{aligned}
& \boldsymbol{q}^{-}-\boldsymbol{q}^{a}=\alpha_{1} \boldsymbol{r}_{1}^{-}, \\
& \boldsymbol{q}^{a}-\boldsymbol{q}^{b}=\alpha_{2} \boldsymbol{r}_{2}^{-}+\alpha_{3} \boldsymbol{r}_{3}^{-}, \\
& \boldsymbol{q}^{c}-\boldsymbol{q}^{d}=\alpha_{7} \boldsymbol{r}_{7}^{+}+\alpha_{8} \boldsymbol{r}_{8}^{+}, \\
& \boldsymbol{q}^{d}-\boldsymbol{q}^{+}=\alpha_{9} \boldsymbol{r}_{9}^{+} .
\end{aligned}
$$

Thus the upwind numerical flux $\left(\boldsymbol{A}_{n} \boldsymbol{q}\right)^{*}$ is defined as (Hesthaven and Warburton, 2007)

$$
\boldsymbol{n} \cdot\left((\boldsymbol{A q})^{*}-\boldsymbol{A}^{-} \boldsymbol{q}^{-}\right)=\alpha_{1} c_{P}^{-} \boldsymbol{Q}^{-} \boldsymbol{r}_{1}^{-}+\alpha_{2} c_{S}^{-} \boldsymbol{Q}^{-} \boldsymbol{r}_{2}^{-}+\alpha_{3} c_{S}^{-} \boldsymbol{Q}^{-} \boldsymbol{r}_{3}^{-}
$$


The expression of $\alpha_{1}, \alpha_{2}$ and $\alpha_{3}$ is derived in detail by Wilcox et al. (Wilcox et al., 2010). After substituting $\alpha_{1}, \alpha_{2}$ and $\alpha_{3}$ in (20), we recover

$$
\begin{aligned}
& \boldsymbol{n} \cdot\left((\boldsymbol{A q})^{*}-\boldsymbol{A}^{-} \boldsymbol{q}^{-}\right)=\frac{\boldsymbol{n} \cdot \llbracket \boldsymbol{S} \rrbracket+\rho^{+} c_{p}^{+} \llbracket \boldsymbol{v} \rrbracket}{\rho^{+} c_{P}^{+}+\rho^{-} c_{P}^{-}}\left[\begin{array}{c}
\lambda^{-} \boldsymbol{I}+2 \mu^{-} \boldsymbol{n} \otimes \boldsymbol{n} \\
\rho^{-} c_{P}^{-} \boldsymbol{n}
\end{array}\right] \\
&-\frac{1}{\left(\rho^{-} c_{S}^{-}+\rho^{+} c_{S}^{+}\right)}\left[\begin{array}{c}
2 \mu^{-} \operatorname{sym}(\boldsymbol{n} \otimes(\boldsymbol{n} \times(\boldsymbol{n} \times \llbracket \boldsymbol{S} \rrbracket))) \\
\rho^{-} c_{S}^{-} \boldsymbol{n} \times(\boldsymbol{n} \times \llbracket \boldsymbol{S} \rrbracket) \\
\end{array}\right. \\
&-\frac{\rho^{+} c_{S}^{+}}{\rho^{-} c_{S}^{-}+\rho^{+} c_{S}^{+}}\left[\begin{array}{c}
2 \mu^{-} \operatorname{sym}(\boldsymbol{n} \otimes(\boldsymbol{n} \times(\boldsymbol{n} \times[\boldsymbol{v}]))) \\
\rho^{-} c_{S}^{-} \boldsymbol{n} \times(\boldsymbol{n} \times[\boldsymbol{v}])
\end{array}\right],
\end{aligned}
$$

where $\boldsymbol{S}=\left[\begin{array}{cc}\sigma_{x x} & \sigma_{x y} \\ & \\ \sigma_{x y} & \sigma_{y y}\end{array}\right]$

\section{Acoustic-acoustic interface}

Acoustic-acoustic interfaces are characterized by $\mu^{-}=\mu^{+}=0$ and thus $\boldsymbol{q}$ reduces to $\hat{\boldsymbol{q}}=\left[\sigma_{x x}, \sigma_{y y}, v_{1}, v_{2}\right]$. We can solve the Riemann problem to compute $\hat{\boldsymbol{q}}$ and keep the number of variables the same as in the case of the elastic-elastic interface, by using relation $\frac{\partial \sigma_{x y}}{\partial t}=$ $\mu\left(\frac{\partial v_{1}}{\partial x_{2}}+\frac{\partial v_{2}}{\partial x_{1}}\right)$. Rankine-Hugonite jump conditions for acoustic-acoustic interface are given 
as

$$
\begin{array}{r}
c_{p}^{-} \boldsymbol{Q}^{-}\left(\boldsymbol{q}^{-}-\boldsymbol{q}^{a}\right)+\boldsymbol{A}_{n}^{-}\left(\boldsymbol{q}^{-}-\boldsymbol{q}^{a}\right)=0 \\
\boldsymbol{A}_{n}^{-} \boldsymbol{q}^{a}-\boldsymbol{A}_{n}^{+} \boldsymbol{q}^{b}=0 \\
-c_{p}^{+} \boldsymbol{Q}^{+}\left(\boldsymbol{q}^{b}-\boldsymbol{q}^{+}\right)+\boldsymbol{A}_{n}^{+}\left(\boldsymbol{q}^{b}-\boldsymbol{q}^{+}\right)=0 .
\end{array}
$$

Now, following the same approach as in the elastic-elastic case, the flux for the acousticacoustic interface is

$$
\boldsymbol{n} \cdot\left((\boldsymbol{A q})^{*}-\boldsymbol{A}^{-} \boldsymbol{q}^{-}\right)=\frac{\boldsymbol{n} \cdot \llbracket \boldsymbol{S} \rrbracket+\rho^{+} c_{p}^{+} \llbracket \boldsymbol{v} \rrbracket}{\rho^{+} c_{P}^{+}+\rho^{-} c_{P}^{-}}\left[\begin{array}{c}
\lambda^{-} \boldsymbol{I} \\
\rho^{-} c_{P}^{-} \boldsymbol{n}
\end{array}\right]
$$

184 where $\boldsymbol{S}=\left[\begin{array}{cc}\sigma_{x x} & 0 \\ 0 & \sigma_{y y}\end{array}\right]$.

\section{Elastic-acoustic and acoustic-elastic interfaces}

For the elastic-acoustic interface $\left(\mu^{-} \neq 0\right.$ and $\left.\mu^{+}=0\right)$, the solutions to the Riemann problem satisfy the Rankine-Hugonite jump conditions:

$$
\begin{aligned}
c_{p}^{-} \boldsymbol{Q}^{-}\left(\boldsymbol{q}^{-}-\boldsymbol{q}^{a}\right)+\boldsymbol{A}_{n}^{-}\left(\boldsymbol{q}^{-}-\boldsymbol{q}^{a}\right) & =0 \\
c_{s}^{-} \boldsymbol{Q}^{-}\left(\boldsymbol{q}^{a}-\boldsymbol{q}^{b}\right)+\boldsymbol{A}_{n}^{-}\left(\boldsymbol{q}^{a}-\boldsymbol{q}^{b}\right) & =0 \\
\boldsymbol{A}_{n}^{-} \boldsymbol{q}^{b}-\boldsymbol{A}_{n}^{+} \boldsymbol{q}^{c} & =0 \\
-c_{p}^{+} \widehat{\boldsymbol{Q}}^{+}\left(\widehat{\boldsymbol{q}}^{c}-\widehat{\boldsymbol{q}}^{+}\right)+\hat{\boldsymbol{A}}_{n}^{+}\left(\widehat{\boldsymbol{q}}^{c}-\widehat{\boldsymbol{q}}^{+}\right) & =0
\end{aligned}
$$


where $\hat{\boldsymbol{q}}=\left[\sigma_{x x}, \sigma_{z z}, v_{1}, v_{2}\right]^{\mathrm{T}}$ and $\widehat{\boldsymbol{Q}}=\left.\boldsymbol{Q}\right|_{\mu=0}$. The resulting numerical flux incorporates an upwind term from an incoming $c_{p}$ characteristics and a boundary term from an incoming $c_{s}$ characteristics (Wilcox et al., 2010). The boundary condition for an incoming $c_{s}$ characteristics is expressed as (Wilcox et al., 2010)

$$
\boldsymbol{s} \cdot \boldsymbol{v}^{-}-2 c_{s}^{-} \boldsymbol{s} . \boldsymbol{S n}=\boldsymbol{s} . \boldsymbol{v}^{+}
$$

where $\boldsymbol{s}$ is the tangential vector along the interface. Solving equations (26)-(29) as in the elastic-elastic case and imposing the boundary condition (30) weakly, the numerical flux for the elastic-acoustic interface is

$$
\begin{aligned}
\boldsymbol{n} \cdot\left((\boldsymbol{A} \boldsymbol{q})^{*}-\boldsymbol{A}^{-} \boldsymbol{q}^{-}\right) & =\frac{\boldsymbol{n} \cdot \llbracket \boldsymbol{S} \rrbracket+\rho^{+} c_{p}^{+} \llbracket \boldsymbol{v} \rrbracket}{\rho^{+} c_{P}^{+}+\rho^{-} c_{P}^{-}}\left[\begin{array}{c}
\lambda^{-} \boldsymbol{I}+2 \mu^{-} \boldsymbol{n} \otimes \boldsymbol{n} \\
\rho^{-} c_{P}^{-} \boldsymbol{n}
\end{array}\right] \\
& -\frac{1}{\rho^{-} c_{S}^{-}}\left[\begin{array}{c}
2 \mu^{-} \operatorname{sym}(\boldsymbol{n} \otimes(\boldsymbol{n} \times(\boldsymbol{n} \times \llbracket \boldsymbol{S} \rrbracket))) \\
\rho^{-} c_{S}^{-} \boldsymbol{n} \times(\boldsymbol{n} \times \llbracket \boldsymbol{S} \rrbracket)
\end{array}\right]
\end{aligned}
$$

Equation (31) could be interpreted as an elastic-elastic flux (21) for $\mu^{+}=0$. Using the same logic, the flux for the acoustic-elastic interface $\left(\mu^{-}=0\right.$ and $\left.\mu^{+} \neq 0\right)$ is

$$
\boldsymbol{n} \cdot\left((\boldsymbol{A} \boldsymbol{q})^{*}-\boldsymbol{A}^{-} \boldsymbol{q}^{-}\right)=\frac{\boldsymbol{n} \cdot \llbracket \boldsymbol{S} \rrbracket+\rho^{+} c_{p}^{+} \llbracket \boldsymbol{v} \rrbracket}{\rho^{+} c_{P}^{+}+\rho^{-} c_{P}^{-}}\left[\begin{array}{c}
\lambda^{-} \boldsymbol{I} \\
\rho^{-} c_{P}^{-} \boldsymbol{n}
\end{array}\right] .
$$


The flux for the acoustic-elastic interface (32) is the same as that of the acoustic-acoustic interface (25). Thus, for the explicit scheme, the flux given in (21), (25), (31) and (32), is appropriately chosen by evaluating the values of $\mu^{-}$and $\mu^{+}$of the medium.

\section{B. Implicit scheme}

The implicit scheme requires $\mu=0$ in the numerical scheme. The $\mathrm{dG}$ scheme must be consistent locally as well as globally. The solid-solid, fluid-fluid, and solid-fluid boundary conditions are expressed as

$$
\begin{gathered}
\boldsymbol{v}^{+}-\boldsymbol{v}^{-}=0 \text { and } \boldsymbol{n} \cdot \boldsymbol{\sigma}^{+}-\boldsymbol{n} \cdot \boldsymbol{\sigma}^{-}=0 \text { on } \Gamma_{\mathrm{SS}} \\
\boldsymbol{n} \cdot\left(\boldsymbol{v}^{ \pm}-\widehat{\boldsymbol{v}}^{\mp}\right)=0 \text { and } \boldsymbol{n} \cdot \boldsymbol{\sigma}^{ \pm}-\boldsymbol{n} \cdot \widehat{\boldsymbol{\sigma}}^{\mp}=0 \text { on } \Gamma_{\mathrm{SF}} \text { and } \Gamma_{\mathrm{FS}} \\
\widehat{\boldsymbol{v}}^{+}-\widehat{\boldsymbol{v}}^{-}=0 \text { and } \widehat{\boldsymbol{\sigma}}^{+}-\widehat{\boldsymbol{\sigma}}^{-}=0 \text { on } \Gamma_{\mathrm{FF}} .
\end{gathered}
$$

To derive the implicit scheme, the matrix form of the acoustic wave equation is required and can be expressed as

$$
\widehat{\boldsymbol{Q}} \frac{\partial \widehat{\boldsymbol{q}}}{\partial t}+\nabla \cdot(\widehat{\boldsymbol{A}} \widehat{\boldsymbol{q}})=\widehat{\boldsymbol{f}}
$$

190 where $\widehat{\boldsymbol{Q}}=\left[\begin{array}{c|c}\lambda & 0 \\ \hline & \\ \hline & \rho \mathbf{I}_{3 \times 3}\end{array}\right], \widehat{\boldsymbol{q}}=\left[\widehat{\sigma}, v_{1}, v_{2}\right]$ with $\widehat{\boldsymbol{\sigma}}=-\lambda \widehat{E}$, where $\widehat{E}$ is the longitudinal strain.

The dG scheme in strong form for (34) is

$$
\begin{gathered}
\int_{D^{k}} \widehat{\boldsymbol{Q}} \frac{\partial \widehat{\boldsymbol{q}}}{\partial t} \cdot \widehat{\boldsymbol{p}} d \boldsymbol{x}+\int_{D^{k}}(\nabla \cdot \widehat{\boldsymbol{A}} \widehat{\boldsymbol{q}}) \cdot \widehat{\boldsymbol{p}} d x+\int_{\partial D^{k}}\left(\boldsymbol{n} \cdot\left((\widehat{\boldsymbol{A}} \widehat{\boldsymbol{q}})^{*}-\widehat{\boldsymbol{A}}^{-} \widehat{\boldsymbol{q}}^{-}\right)\right) \cdot \widehat{\boldsymbol{p}} d \boldsymbol{x} \\
=\int_{D^{k}} \widehat{\boldsymbol{f}} \cdot \widehat{\boldsymbol{p}} d \boldsymbol{x} \text { for all } \widehat{\boldsymbol{p}} \in \boldsymbol{V}_{h}\left(\Omega_{h}\right),
\end{gathered}
$$


To derive an implicit scheme, we consider a Lax-Friedrich flux (Hesthaven and Warburton, 2007), i.e.,

$$
\boldsymbol{n} \cdot\left(\boldsymbol{A} \boldsymbol{q}^{*}\right)=n_{x}\{\{\boldsymbol{A} \boldsymbol{q}\}\}+n_{y}\{\{\boldsymbol{A} \boldsymbol{q}\}\}+\frac{c_{\max }}{2}[\boldsymbol{q}]
$$

where $c_{\max }$ is the maximum phase velocity of the modes present in the system.

Substituting (36) in (10), the surface term is recovered as

$$
\boldsymbol{n} \cdot\left((\boldsymbol{A} \boldsymbol{q})^{*}-\boldsymbol{A}^{-} \boldsymbol{q}^{-}\right)=\frac{1}{2} \llbracket \boldsymbol{A} \boldsymbol{q} \rrbracket+\frac{c_{\max }}{2}[\boldsymbol{q}] .
$$

In (37), the first term, $\frac{1}{2} \llbracket \boldsymbol{A q} \rrbracket$ is responsible for the interface boundary conditions explained in $(33 \mathrm{a})-(33 \mathrm{c})$, whereas the second term $\frac{c_{\max }}{2}[\boldsymbol{q}]$ is a penalty term that stabilizes the scheme. Implementation of the flux in (36) for the acousto-elastic system with $\mu=0$, will produce the wrong results as the numerical scheme in (10) with the flux in (37) is consistent for purely elastic or acoustic systems, but not for a coupled acoustic-elastic system (Wilcox et al., 2010).

To accurately incorporate the interface conditions at solid-solid, fluid-fluid, solid-fluid and fluid-solid boundaries in (10) and (35), we express the dG formulation for the acoustic and elastic conditions as follows

$$
\begin{aligned}
& \int_{D_{S}^{k}} \boldsymbol{Q} \frac{\partial \boldsymbol{q}}{\partial t} \cdot \boldsymbol{p} d \boldsymbol{x}+\int_{D_{S}^{k}}(\nabla \cdot \boldsymbol{A} \boldsymbol{q}) \cdot \boldsymbol{p} d \boldsymbol{x}+\frac{1}{2} \int_{\partial D_{S S}^{k}} \llbracket \boldsymbol{A}_{n} \boldsymbol{q} \rrbracket_{S S} \cdot \boldsymbol{p} d \boldsymbol{x}+\frac{1}{2} \int_{\partial D_{S F}^{k}} \llbracket \boldsymbol{A}_{n} \boldsymbol{q} \rrbracket_{S F} \cdot \boldsymbol{p} d \boldsymbol{x} \\
& =\int_{D^{k}} \boldsymbol{f} \cdot \boldsymbol{p} d \boldsymbol{x} \text { for all } \boldsymbol{p} \in \boldsymbol{V}_{h}\left(\Omega_{h}\right), \\
& \int_{D_{F}^{k}} \widehat{\boldsymbol{Q}} \frac{\partial \widehat{\boldsymbol{q}}}{\partial t} \cdot \widehat{\boldsymbol{p}} d \boldsymbol{x}+\int_{D_{F}^{k}}(\nabla \cdot \widehat{\boldsymbol{A}} \widehat{\boldsymbol{q}}) \cdot \widehat{\boldsymbol{p}} d \boldsymbol{x}+\frac{1}{2} \int_{\partial D_{F F}^{k}} \llbracket \widehat{\boldsymbol{A}_{n}} \widehat{\boldsymbol{q}} \rrbracket_{F F} \cdot \widehat{\boldsymbol{p}} d \boldsymbol{x}+\frac{1}{2} \int_{\partial D_{F S}^{k}} \llbracket \widehat{\boldsymbol{A}_{n}} \widehat{\boldsymbol{q}} \rrbracket_{F S} \cdot \widehat{\boldsymbol{p}} d \boldsymbol{x} \\
& =\int_{D_{F}^{k}} \widehat{\boldsymbol{f}} \cdot \widehat{\boldsymbol{p}} d \boldsymbol{x} \text { for all } \widehat{\boldsymbol{p}} \in \boldsymbol{V}_{h}\left(\Omega_{h}\right),
\end{aligned}
$$


where we define

$$
\begin{aligned}
\llbracket \boldsymbol{A}_{n} q \rrbracket_{S S} & =\left(\boldsymbol{A}_{n} \boldsymbol{q}\right)^{+}-\left(\boldsymbol{A}_{n} \boldsymbol{q}\right)^{-}, \quad \llbracket \boldsymbol{A}_{n} \boldsymbol{q} \rrbracket_{S F}=\boldsymbol{T}^{T}(\widehat{\boldsymbol{A}} \widehat{\boldsymbol{q}})^{+}-\left(\boldsymbol{A}_{n} \boldsymbol{q}\right)^{-} \\
\llbracket \widehat{\boldsymbol{A}}_{n} \widehat{\boldsymbol{q}} \rrbracket_{F F} & =\left(\widehat{\boldsymbol{A}}_{n} \widehat{\boldsymbol{q}}\right)^{+}-\left(\widehat{\boldsymbol{A}}_{n} \widehat{\boldsymbol{q}}\right)^{-}, \quad \llbracket \widehat{\boldsymbol{A}}_{n} \widehat{\boldsymbol{q}} \rrbracket_{F S}=\boldsymbol{T}\left(\boldsymbol{A}_{n} \boldsymbol{q}\right)^{+}-\left(\widehat{\boldsymbol{A}}_{n} \widehat{\boldsymbol{q}}\right)
\end{aligned}
$$

with the map $T: \mathbb{R}^{5} \rightarrow \mathbb{R}^{2}$ given by (Ye et al., 2016)

$$
T \boldsymbol{q}=\left[\begin{array}{c}
\boldsymbol{n} \cdot \boldsymbol{\sigma} \cdot n \\
(\boldsymbol{n} \cdot \boldsymbol{v}) \boldsymbol{n}
\end{array}\right] \quad \text { and } T^{T} \widehat{\boldsymbol{q}}=\left[\begin{array}{c}
(\boldsymbol{n} \boldsymbol{n} \widehat{\sigma}) \\
(\boldsymbol{n} \cdot \widehat{\boldsymbol{v}}) \boldsymbol{n}
\end{array}\right],
$$

198 where

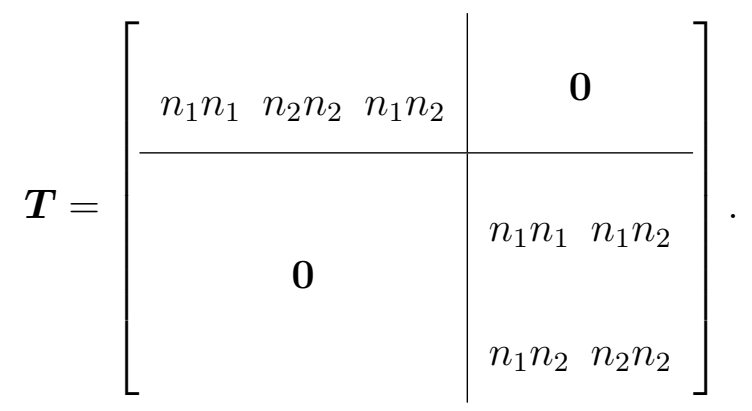

In (38) and (39), the numerical fluxes are central fluxes, which may be unstable. To stabilize these formulations, a penalization is used (Guo et al., 2019; Warburton, 2013; Ye et al., 2016). In this study, we used a penalization method of fluxes proposed by Ye et al.(Warburton, 2013). The penalized flux for the solid-solid is

$$
\begin{gathered}
F_{E}=\frac{1}{2} \int_{\partial D_{S S}^{k}} \llbracket \boldsymbol{A}_{n} \boldsymbol{q} \rrbracket_{S S} \cdot \boldsymbol{p} d \boldsymbol{x}+\frac{1}{2} \int_{\partial D_{S F}^{k}} \llbracket \boldsymbol{A}_{n} \boldsymbol{q} \rrbracket_{S F} \cdot \boldsymbol{p} d \boldsymbol{x}+\frac{\alpha}{2}\left[\int_{\partial D_{S S}^{k}}\left(\boldsymbol{A}_{n}^{T} \llbracket \boldsymbol{A}_{n} \boldsymbol{q} \rrbracket\right) \cdot \boldsymbol{p} d \boldsymbol{x}\right. \\
\left.+\int_{\partial D_{S F}^{k}}\left(\boldsymbol{A}_{n}^{T} \llbracket \boldsymbol{A}_{n} \boldsymbol{q} \rrbracket\right) \cdot \boldsymbol{p} d \boldsymbol{x}\right]
\end{gathered}
$$

where $\alpha>0$. Similarly, the penalized flux for the fluid-fluid and fluid-solid case is

$$
\begin{aligned}
F_{A}=\frac{1}{2} \int_{\partial D_{F F}^{k}} \llbracket \widehat{\boldsymbol{A}_{n}} \widehat{\boldsymbol{q}} \rrbracket_{F F} \cdot \widehat{\boldsymbol{p}} d \boldsymbol{x} & +\frac{1}{2} \int_{\partial D_{F S}^{k}} \llbracket \widehat{\boldsymbol{A}_{n}} \widehat{\boldsymbol{q}} \rrbracket_{F S} \cdot \widehat{\boldsymbol{p}} d \boldsymbol{x}+\frac{\alpha}{2}\left[\int_{\partial D_{F F}^{k}} \widehat{\boldsymbol{A}}_{n}^{T} \llbracket \widehat{\boldsymbol{A}}_{n} \widehat{\boldsymbol{q}} \rrbracket \cdot \boldsymbol{p} d \boldsymbol{x}\right. \\
& \left.+\int_{\partial D_{F S}^{k}} \widehat{\boldsymbol{A}}_{n}^{T} \llbracket \widehat{\boldsymbol{A}}_{n} \widehat{\boldsymbol{q}} \rrbracket \cdot \boldsymbol{p} d \boldsymbol{x}\right]
\end{aligned}
$$


TABLE I. Material properties

\begin{tabular}{|llll|}
\hline Material & $\lambda$ & $\mu$ & $\rho$ \\
& & & \\
& $(\mathrm{GPa})$ & $(\mathrm{GPa})$ & $\left(\mathrm{kg} / \mathrm{m}^{3}\right)$ \\
\hline \hline water & 2.25 & 0 & 1000 \\
plexiglass & 4.3315 & 2.2799 & 1180 \\
glass & & & \\
\hline
\end{tabular}

TABLE II. Velocities of body and surface waves

\begin{tabular}{||cccccc||}
\hline Interface & $c_{f}$ & $c_{L}$ & $c_{S}$ & $c_{S c}$ & $c_{p R}$ \\
& & & & & \\
& $(\mathrm{~m} / \mathrm{s})$ & $(\mathrm{m} / \mathrm{s})$ & $(\mathrm{m} / \mathrm{s})$ & $(\mathrm{m} / \mathrm{s})$ & $(\mathrm{m} / \mathrm{s})$ \\
\hline \hline water-plexiglass & 1500 & 2745 & 1390 & 1058 & 1363 \\
water-glass & 1500 & 5712 & 3356 & 1496 & 3091 \\
\hline
\end{tabular}

\section{Time discretization}

We employ the low-storage explicit Runge-Kutta (LSERK) method (Cockburn and Shu, 2001). The LSERK method is a single-step method but comprises five intermediate stages.

LSERK is preferred over other methods as it saves memory at the cost of computation time. 
A stable CFL condition, depending on the polynomial degree $N$, is derived by Cockburn and Shu (Cockburn and Shu, 2001) and used here.

\section{Forcing function}

The forcing function $\boldsymbol{f}$, in (8), is the product of a Dirac delta in space $\left(\boldsymbol{x}_{0}\right)$ and a Ricker wavelet in time,

$$
\boldsymbol{f}=\delta\left(\boldsymbol{x}-x_{0}\right) h(t)
$$

where $h(t)$ is a wavelet, given by

$$
h(t)=\exp \left[-2 f_{p}^{2}\left(t-t_{0}\right)^{2}\right] \cos \left[2 \pi f_{p}\left(t-t_{0}\right)\right],
$$

with $f_{p}$ being the central peak frequency and $t_{0}=3 /\left(4 f_{p}\right)$. In subsequent sections, $f_{p}$ is taken to be $500 \mathrm{kHz}$ with a dilatational moment force $\left(f_{x x}=f_{z z}\right.$ and $\left.f_{x y}=0\right)$.

\section{COMPUTATIONAL EXPERIMENTS}

To perform the computational experiments, we consider a medium including waterplexiglass and water-glass interfaces. The material properties are given in Table I. The velocities of the body and surface or interface waves, computed from the dispersion relation(6), are given in Table II. The medium defined by the water-plexiglass combination represents the soft-solid system, whereas the water-glass interface is the stiff-solid condition. To determine the existence and evolution of the interface waves in soft and stiff solid conditions, we first solve the coupled acoustic-elastic system using the explicit numerical scheme, 


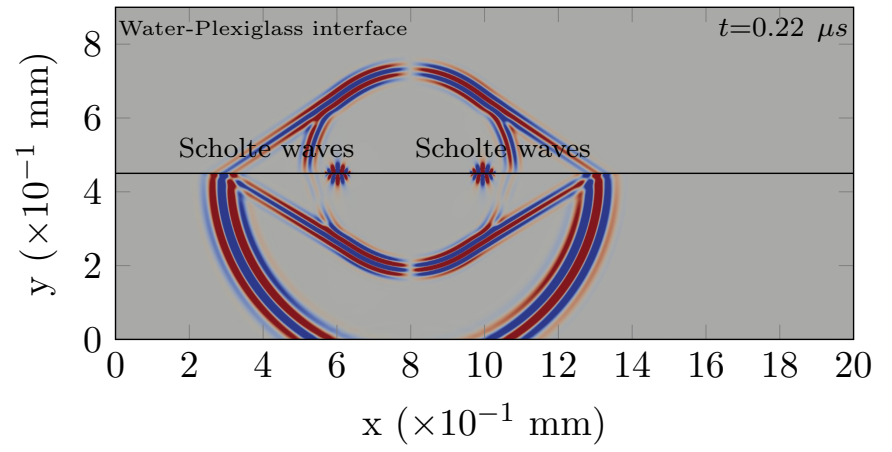

(a) Horizontal particle velocity, $v_{1}$ at $t=0.22 \mu \mathrm{s}$

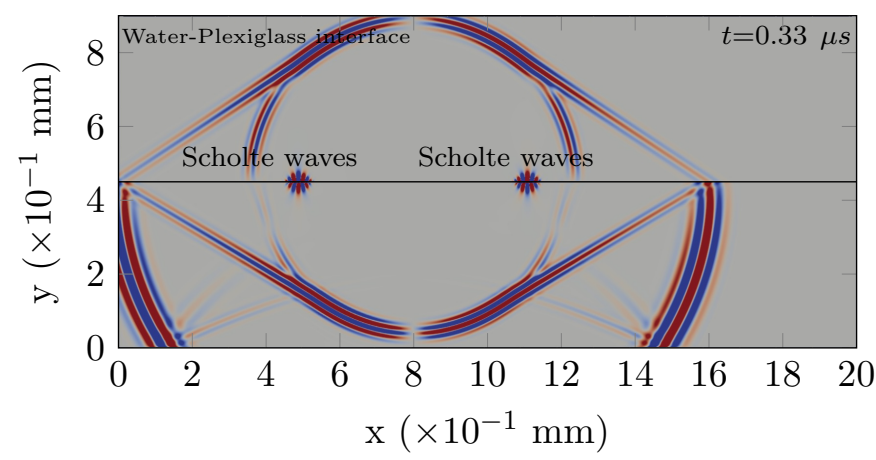

(c) Horizontal particle velocity, $v_{1}$ at $t=0.33 \mu \mathrm{s}$

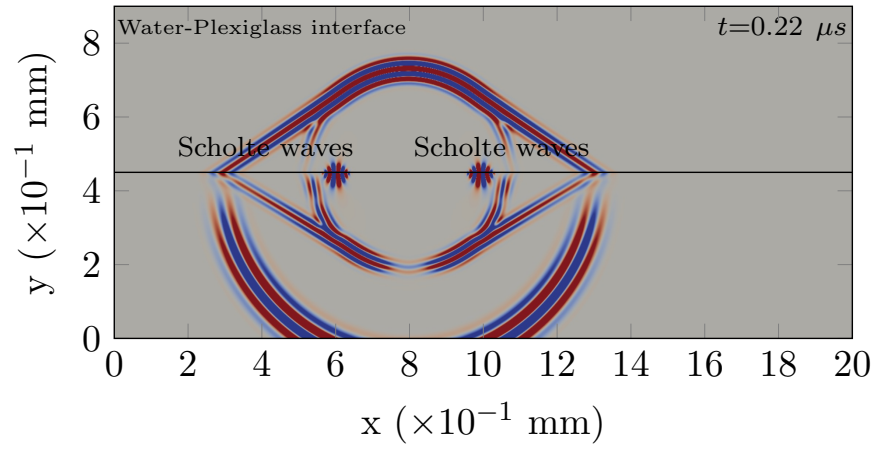

(b) Vertical particle velocity, $v_{3}$ at $t=0.22 \mu \mathrm{s}$

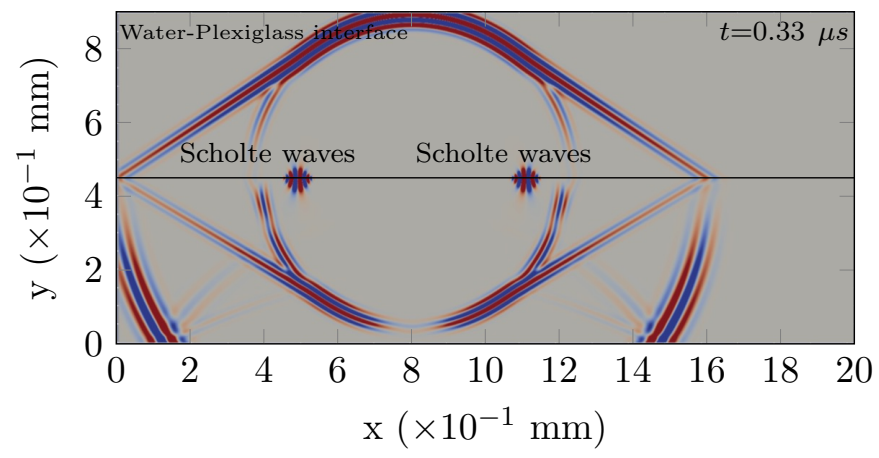

(d) Vertical solid particle velocity, $v_{3}$ at $t=0.33 \mu \mathrm{s}$

FIG. 2. Snapshots of the simulated interface waves for a water-plexiglass interface, using explicit boundary-conditions, which shows the horizontal and vertical particle velocities at $0.22 \mu \mathrm{s}(\mathrm{a}, \mathrm{b})$ and $0.33 \mu \mathrm{s}(\mathrm{c}, \mathrm{d})$. The source is located in the solid at $38.4 \mu \mathrm{m}$ below the interface, and it is an explosion $(f x x=f z z)$, with a central frequency of $500 \mathrm{kHz}$.

218

defined in (10) in subsection V A. Figure 2 shows snapshots of the particle velocities computed for the water-plexiglass or soft-solid condition. The size of the computational domain is $[0.20 \mathrm{~mm} \times 0.9 \mathrm{~mm}]$. The minimum size of the edges of the equilateral triangles, used to mesh the domain, in the $x$ and $y$ directions, are $0.37 \mathrm{~mm}$ and $0.56 \mathrm{~mm}$, respectively. The degree of polynomial $(N)$ is 4 and the time step $d t$ used for time integration is 0.217 ns. 


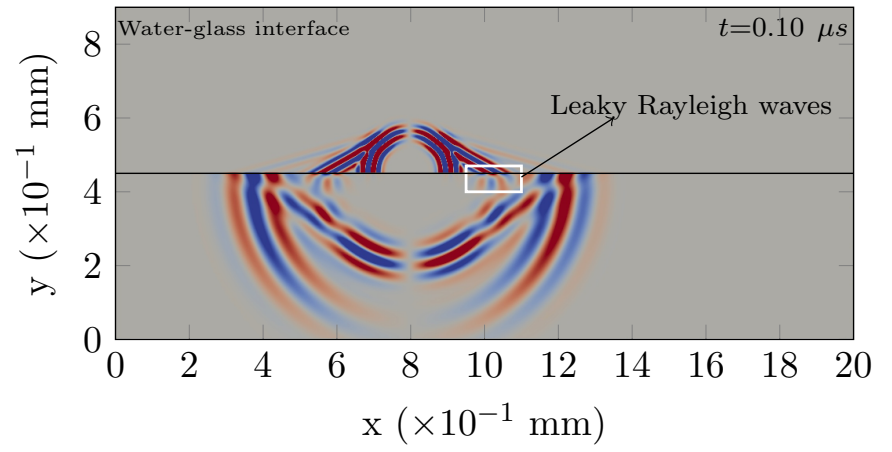

(a) Horizontal particle velocity, $v_{1}$ at $t=0.10 \mu \mathrm{s}$

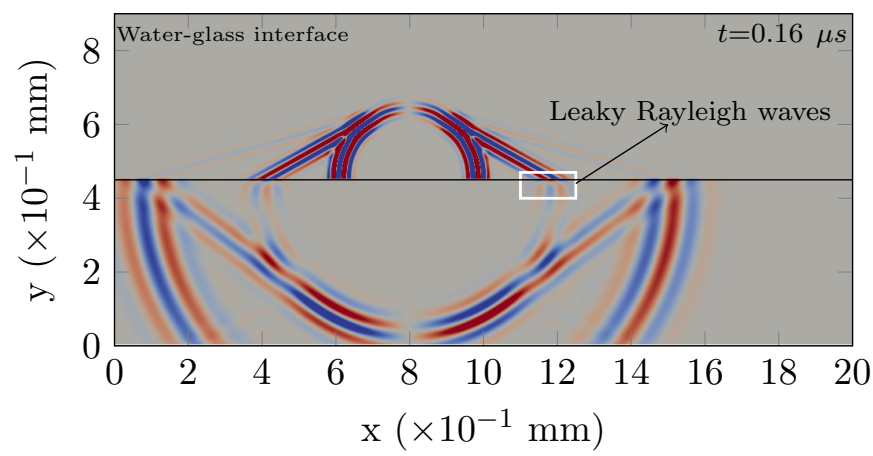

(c) Horizontal particle velocity, $v_{1}$ at $t=0.16 \mu \mathrm{s}$

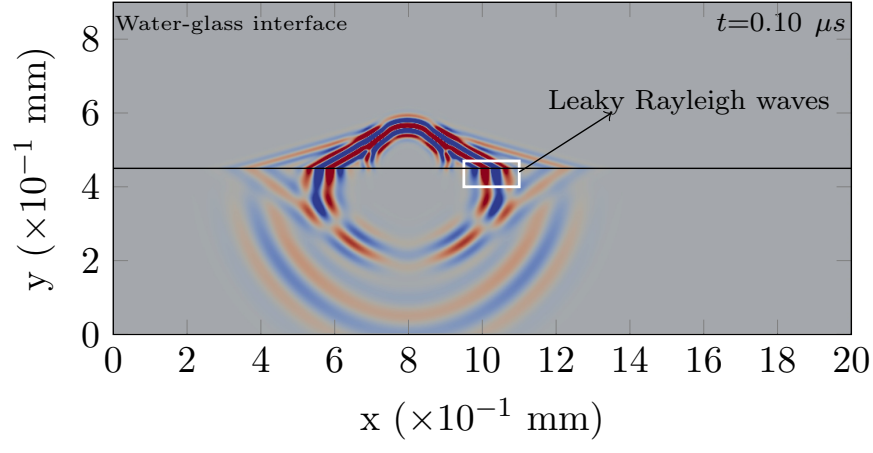

(b) Vertical particle velocity, $v_{3}$ at $t=0.10 \mu \mathrm{s}$

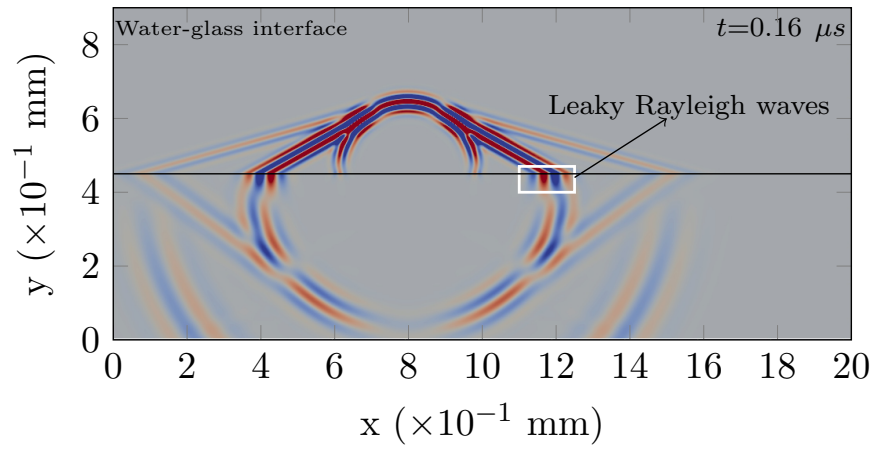

(d) Vertical solid particle velocity, $v_{3}$ at $t=0.16 \mu \mathrm{s}$

FIG. 3. Snapshots of the simulated interface waves for a water-glass interface, using explicit boundary-conditions, which shows the horizontal and vertical particle velocities at $0.10 \mu \mathrm{s}$ (a, b) and $0.16 \mu \mathrm{s}(\mathrm{c}, \mathrm{d})$. The source is located in the solid at $38.4 \mu \mathrm{m}$ below the interface, and it is an explosion $(f x x=f z z)$, with a central frequency of $500 \mathrm{kHz}$. 
Figures $2 \mathrm{a}$ and $\mathrm{b}$ represent the snapshots of the horizontal $\left(v_{1}\right)$ and vertical $\left(v_{2}\right)$ particle velocities at $0.22 \mu \mathrm{s}$. As discussed, the presence of the Scholte wave, is observed and marked in Figures 2a and b. To visualize the evolution of the Scholte wave, Figures 2c and d represent the particle velocities $\left(v_{1}, v_{2}\right)$ at $t=0.33 \mu \mathrm{s}$. The Scholte wave moves to a distance of $1.1 \mathrm{~mm}$ (Figures $2 \mathrm{c}$ and $2 \mathrm{~d}$ ) at $t=0.33 \mu$ s from $0.9836 \mathrm{~mm}$ (Figures $2 \mathrm{a}$ and $2 \mathrm{~b}$ ) measured at $t=0.22 \mu \mathrm{s}$. Thus the velocity of the Scholte wave is equal to $1058.18 \mathrm{~m} / \mathrm{s}$. This in agreement with the velocity computed from the dispersion relation, shown in Table II, with a relative error of $0.017 \%$.

Figure 3 represents the snapshots of the horizontal $\left(v_{1}\right)$ and vertical particle velocities $\left(v_{3}\right)$ for the water-glass system, i.e., a stiff solid medium. The discretization parameters are the same as those used in Figure 1 except $d t$, which is .104 ns. Figures 3a and 3b represent snapshot at $t=0.10 \mu \mathrm{s}$. The presence of a leaky Rayleigh mode is observed (also marked by a white box) at $x=0.1085 \mathrm{~mm}$. To see the evolution of the leaky Rayleigh wave, the snapshots of horizontal and particle velocities computed at $t=0.16 \mu$ s are shown in Figures $3 \mathrm{c}$ and $3 \mathrm{~d}$. The leaky Rayleigh mode is present $x=0.1085 \mathrm{~mm}$. Thus, the velocity of the leaky Rayleigh mode computed is $3091.67 \mathrm{~m} / \mathrm{s}$, with a relative error of $0.022 \%$ as compared to the velocity obtained from the dispersion relation. 
Figures 4-8 show the comparison of the numerical solution against the analytical solution obtained by Berg et al. (Berg et al., 1994) using the method of Cagniard-de Hoop (de Hoop and Van der Hijden, 1983). Figure 4 shows the simulation of the reflection response using an upwind flux (explicit boundary conditions) with a polynomial degree $N=4$. Source and receiver are located in the fluid at $0.0158831 \mathrm{~m}$ above the interface, and are separated by $0.016 \mathrm{~m}$. The source is an explosion with a central frequency of $500 \mathrm{kHz}$. The discretization parameters for the water-plexiglass and water-glass models are the same as those used for results shown in Figures 2 and 3, respectively. The solid line and circles correspond to the analytical and numerical solutions, respectively. Figures $4 \mathrm{a}$ and $4 \mathrm{~b}$ show the $x$ and $z$ components of the normalized particle velocities corresponding to the water-plexiglass model, respectively. Figures $4 \mathrm{c}$ and $4 \mathrm{~d}$ show the $x$ and $z$ components of the normalized particlevelocity corresponding to the water-glass model, respectively. The direct and reflection modes are marked in the figures. The agreement between numerical and analytical solutions for both models is very good. Next, we verify the ability of the numerical scheme to model interface waves. Source and receiver are both located in the solid at $38.4 \mu \mathrm{m}$ with an offset of $0.1 \mathrm{~m}$. The discretization parameters are the same as those in Figure 4. Figure $5 \mathrm{a}$ and $5 \mathrm{~b}$ show a comparison of the $x$ and $z$ components of the normalized particle velocities for the water-plexiglass model (soft solid condition). The solutions show a very good agreement. The dominance of the Scholte wave in both components is clearly observed. A comparison of the analytical and numerical solutions for the water-glass model (stiff-solid system) is shown in Figures 5c and 5d. The agreement between the solutions is satisfactory. The 


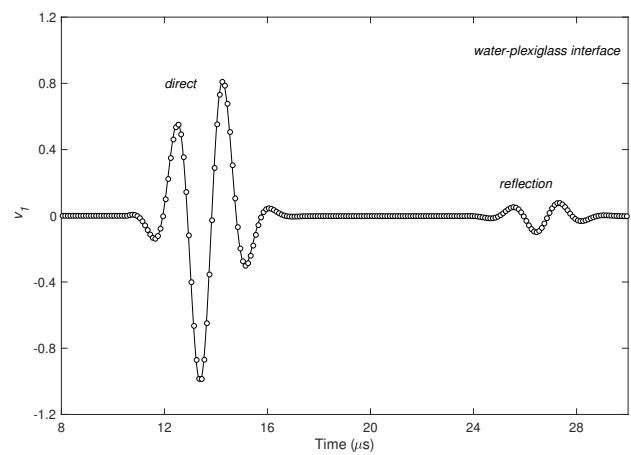

(a) $x$-component. Water-plexiglass interface

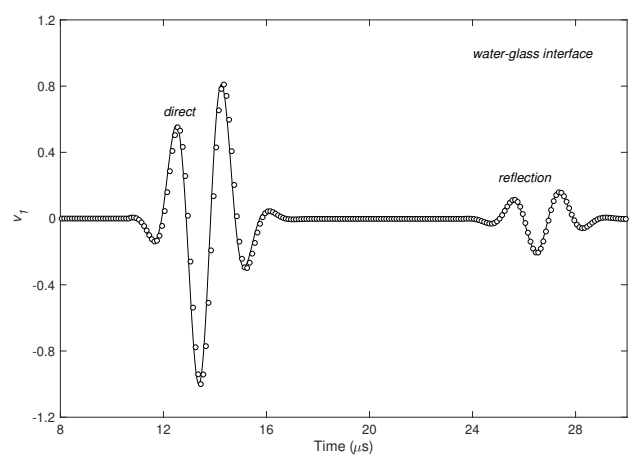

(c) $x$-component. Water-glass interface

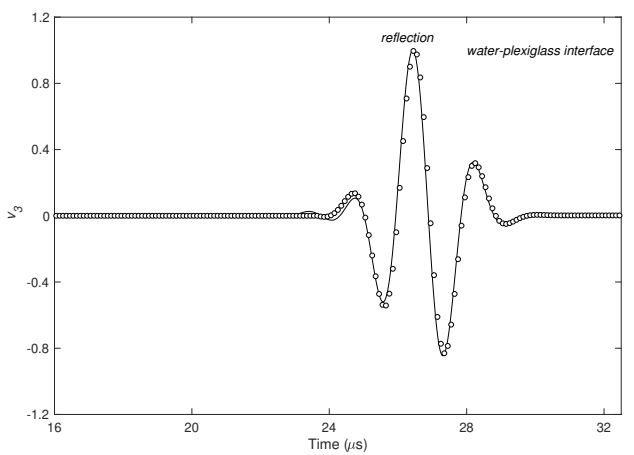

(b) z-component. Water-plexiglass interface

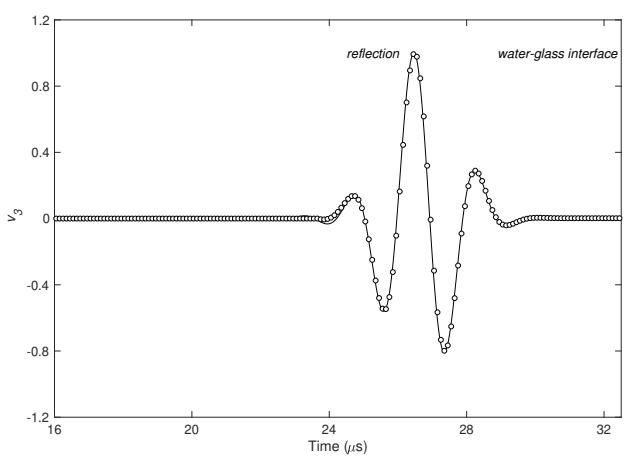

(d) z component. Water-glass interface

FIG. 4. Explicit boundary-condition: Simulation of the reflection response using an upwind flux, where the solid line and circles correspond to the analytical and numerical solutions, respectively. The figure shows the normalized particle-velocity components corresponding to water-plexiglass (a and b) and water-glass (c and d). Source and receiver are located in the fluid at $0.0158831 \mathrm{~m}$ above the interface, and are separated by $0.016 \mathrm{~m}$. The source is an explosion, with a central frequency of $500 \mathrm{kHz}$. 


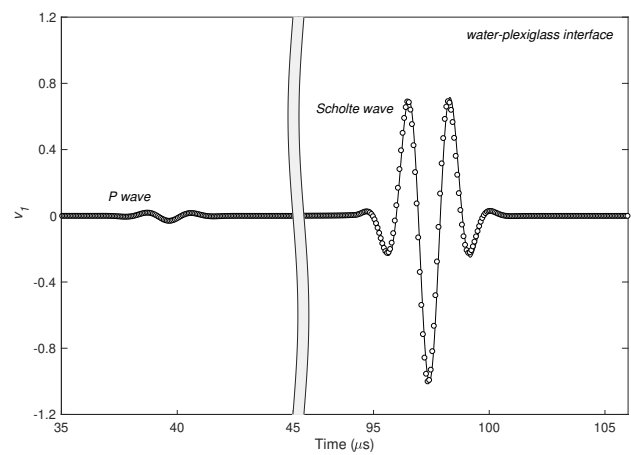

(a) $x$-component. Water-plexiglass interface

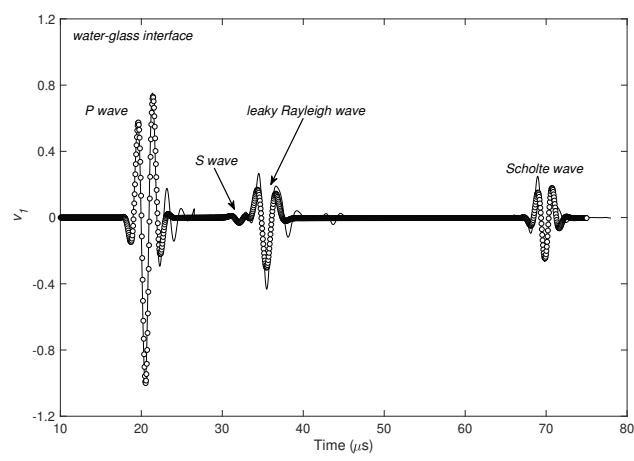

(c) $x$-component. Water-glass interface

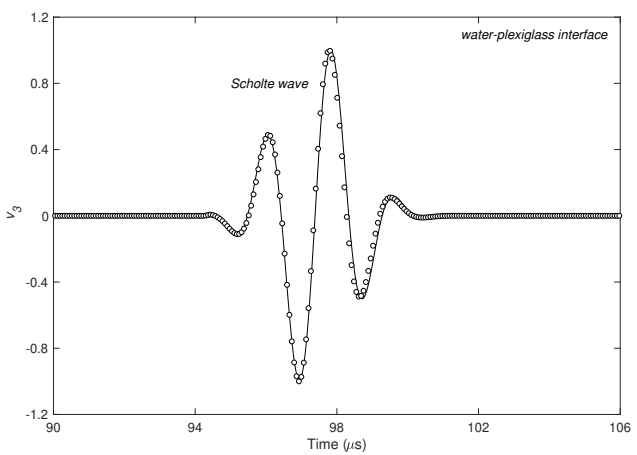

(b) z-component. Water-plexiglass interface

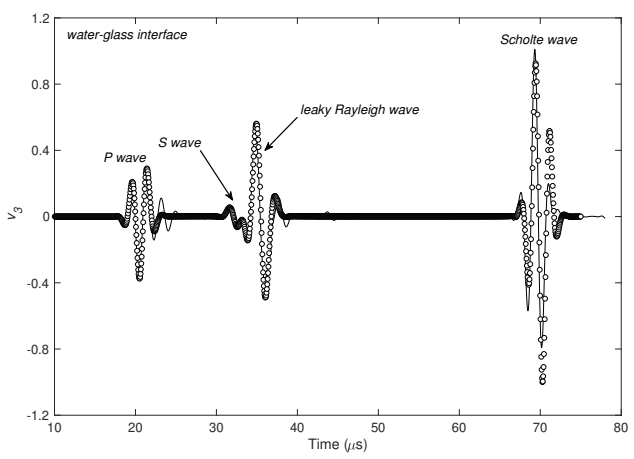

(d) z-component. Water-glass interface

FIG. 5. Explicit boundary-condition: Simulation of interface-waves, where the solid line and dots correspond to the analytical and numerical solutions, respectively. The figure shows the normalized particle-velocity components corresponding to water-plexiglass (a and b) and water-glass (c and d). Source and receiver are both located in the solid at $38.4 \mu \mathrm{m}$ below the interface, with a separation $0.1 \mathrm{~m}$. The source is an explosion $(f x x=f z z)$, with a central frequency of $500 \mathrm{kHz}$. 


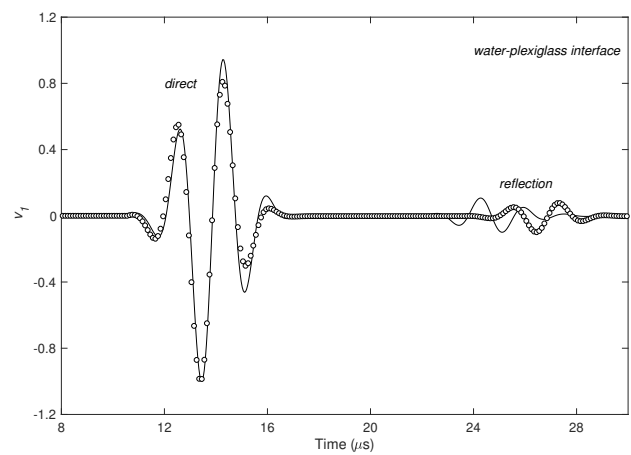

(a) $x$ component. Water-plexiglass interface

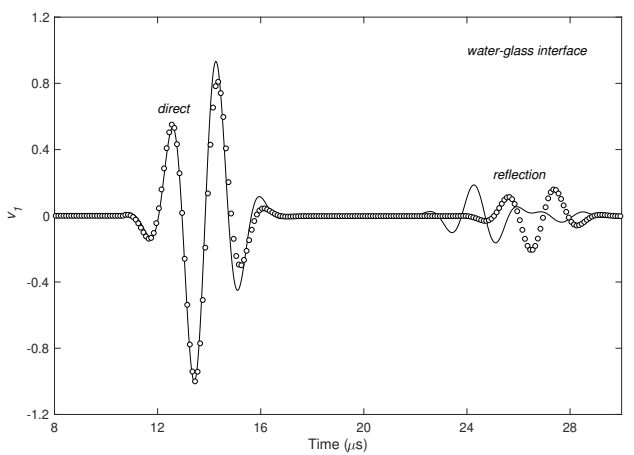

(b) $z$ component. Water-plexiglass interface

FIG. 6. Implicit boundary-condition: Simulation of the reflection response using the Lax-Friedrich (inconsistent) flux, where the solid line and circles correspond to the analytical and numerical solutions, respectively. The figure shows the normalized particle-velocity components corresponding to water-plexiglass ( $\mathrm{a}$ and $\mathrm{b}$ ). Source and receiver are located in the fluid at $0.0158831 \mathrm{~m}$ above the interface, and are separated by $0.016 \mathrm{~m}$. The source is an explosion, with a central frequency of $500 \mathrm{kHz}$

presence of leaky Rayleigh and Scholte waves is clearly observed, along with the P and S waves. Next, we show the ability of the implicit numerical scheme with Lax-Freedrich flux (37) and setting the $\mu=0$. Figures $6 \mathrm{a}$ and $6 \mathrm{~b}$ show a comparison between the numerical and analytical solutions of the particle velocities for the water-plexiglass and water-glass models, respectively. It is clear that the reflection mode is not accurately simulated by the scheme. This results from the scheme not being consistent.

Now, we show results of the numerical scheme using the penalty fluxes expressed in (41) and (42). Figures 7 and 8 show the comparison of the analytical and numerical solutions 


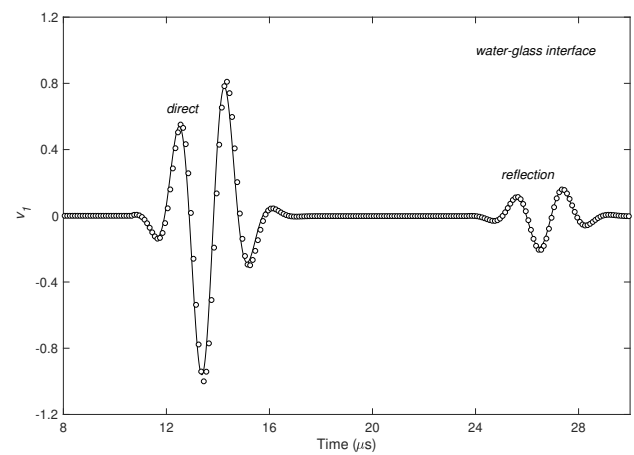

(a) $x$ component. Water-plexiglass interface

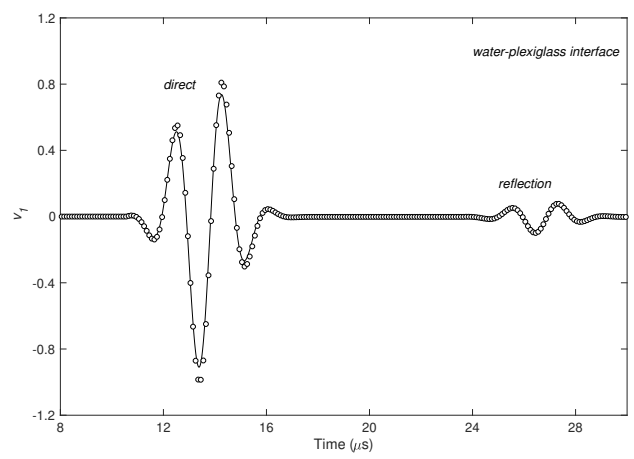

(c) $x$ component. Water-plexiglass interface

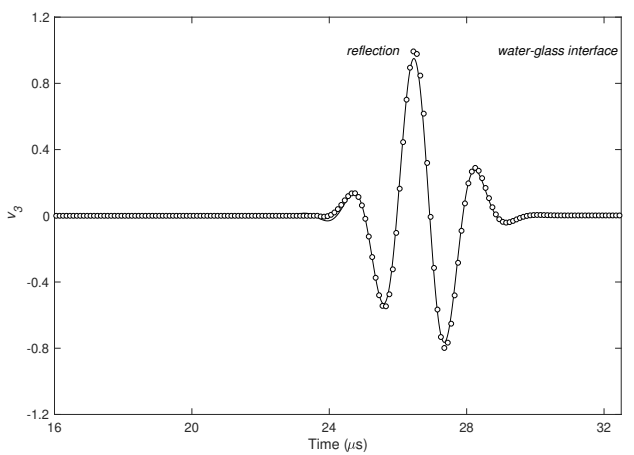

(b) z component. Water-plexiglass interface

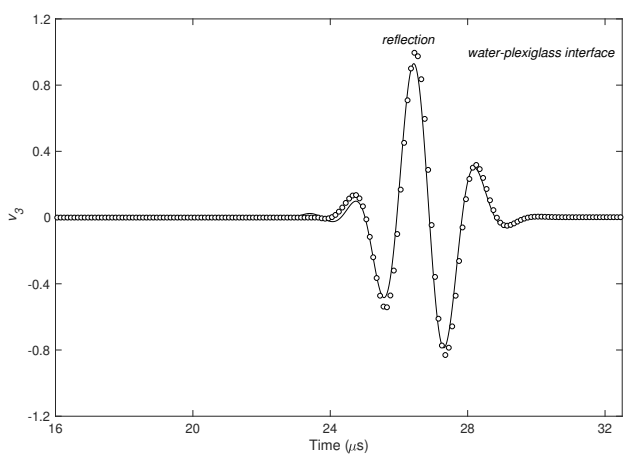

(d) z component. Water-plexiglass interface

FIG. 7. Implicit boundary-condition: Simulation of the reflection response using the penalized consistent central flux, where the solid line and circles correspond to the analytical and numerical solutions, respectively. The figure shows the normalized particle-velocity components corresponding to water-plexiglass ( $\mathrm{a}$ and b) and water-glass (c and d). Source and receiver are located in the fluid at $0.0158831 \mathrm{~m}$ above the interface, and are separated by $0.016 \mathrm{~m}$. The source is an explosion, with a central frequency of $500 \mathrm{kHz}$ 


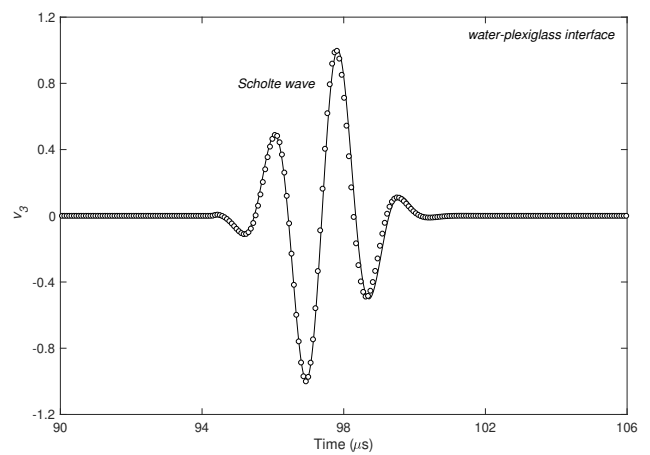

(a) $x$-component. Water-plexiglass interface

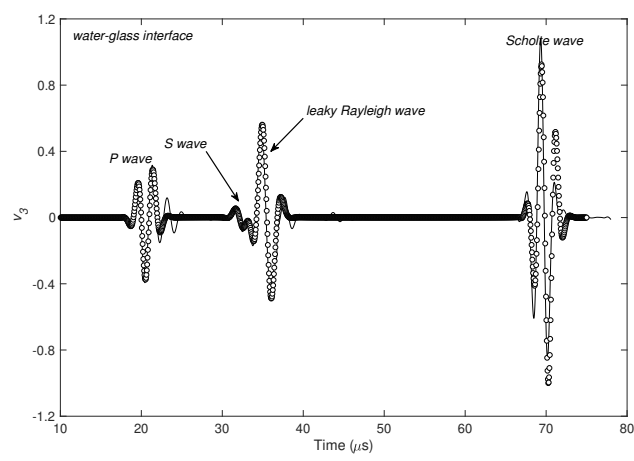

(c) $x$-component. Water-glass interface

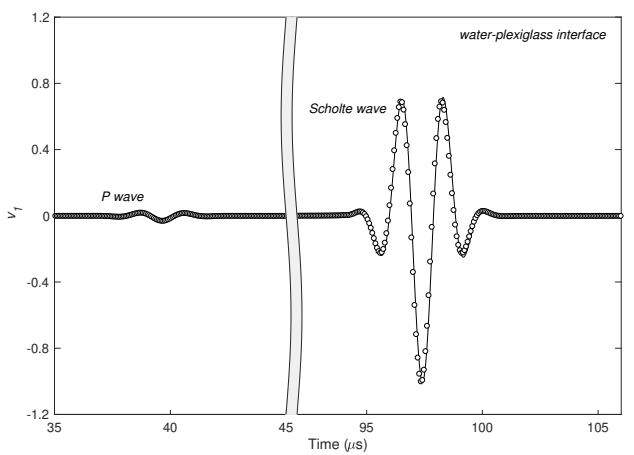

(b) z-component. Water-plexiglass interface

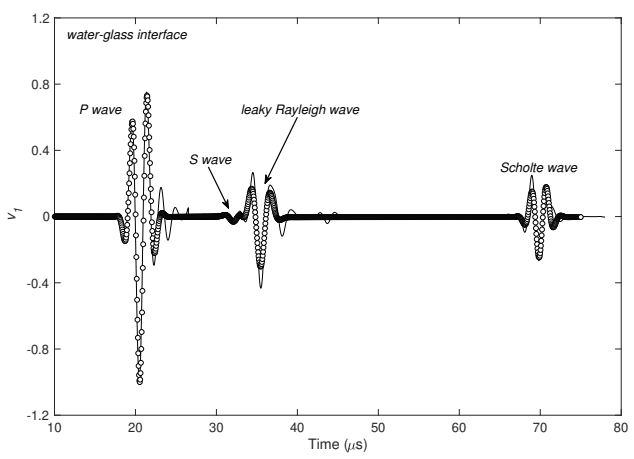

(d) z-component. Water-glass interface

FIG. 8. Implicit boundary-condition: Simulation of interface-waves using a penalized consistent central flux, where the solid line and dots correspond to the analytical and numerical solutions, respectively. The figure shows the normalized particle-velocity components corresponding to waterplexiglass ( $\mathrm{a}$ and b) and water-glass (c and d). Source and receiver are both located in the solid at $38.4 \mu \mathrm{m}$ below the interface, with a separation $0.1 \mathrm{~m}$. The source is an explosion $(f x x=f z z)$, with a central frequency of $500 \mathrm{kHz}$. 
for the reflection and interface problems. Discretization parameters and source-receiver geometry are same as those used for the results shown in Figure 4 and 5. Figures 6 and 7 show a very good agreement between the numerical and analytical solutions for the body ( $\mathrm{P}$ and $\mathrm{S}$ waves) and interface waves, confirming that the implicit scheme is suitable provided a consistent flux is used.

\section{CONCLUSIONS}

To our knowledge, past works dealing with the simulation of waves at fluid-solid interfaces, performed with the finite-element method (continuous or discontinuous Galerkin) with unstructured grids, do not show the complete set of waves (i.e. including the Scholte and leaky-Rayleigh waves) and a comparison with the analytical solution has not been performed. This test is essential to verify the correct simulation of wave propagation in the presence of interfaces, corresponding to soft and stiff fluid-solid systems. Here, we present analytic and numerical results for the simulation of waves propagating at the fluid-solid interface for two solids: plexiglass (soft) and glass (stiff). To perform the simulations, we use a discontinuous Galerkin method with upwind and penalty fluxes to implement the correct boundary conditions. We show that setting $\mu=0$ in a $\mathrm{dG}$ formulation, corresponding to elastic wave equation, provides wrong results. The numerical solution obtained from upwind and penalty

flux based methods produce the correct results and are in very good agreement with the analytical solutions. 


\section{ACKNOWLEDGMENTS}

KS would like to thank Dr. Ruichao Ye, ExxonMobil, Houston, TX for his time to discuss the penalty flux and its implementation in the $\mathrm{dG}$ formulation. Author would like to thank BP America, Houston, TX for discussions and guidance on the topic.

Ansell, J. H. (1972). "The roots of the stoneley wave equation for solid-liquid interfaces," Pure and Applied Geophysics 94(1), 172-188.

Ash, E. A., and Paige, E. G. (1985). Rayleigh-Wave Theory and and Application: Proceedings of An International Symposium Organised by the Rank Prize Funds at the Royal Institution, London, 1985 (Springer-Verlag).

Berg, P., If, F., Nielsen, P., and Skovgaard, O. (1994). Analytical reference solutions, in Modeling The Earth For Oil Exploration, (K. Helbig, ed., Pergamon Press), pp. 421-427.

Cagniard, L. (1962). Reflection and Refraction of Progressive Seismic Waves, Translation and Revision of Cagniard, L., 1939, Réflexion et Réfraction des Ondes Séismiques Progressives (McGraw-Hill, NY).

Carbajal-Romero, M., Flores-Mendez, E., Flores-Guzmán, N., Núñez-Farfán, J., OliveraVillaseñor, E., and Sánchez-Sesma, F. J. (2013). "Scholte waves on fluid-solid interfaces by means of an integral formulation," Geofísica Internacional 52(1), 21-30. 
Carcione, J. M. (2014). Wave Fields in Real Media. Theory and numerical simulation of wave propagation in anisotropic, anelastic, porous and electromagnetic media, $3^{\text {rd }}$ edition (Elsevier).

Carcione, J. M., Bagaini, C., Ba, J., Wang, E., and Vesnaver, A. (2018). "Waves at fluidsolid interfaces: explicit versus implicit formulation of the boundary condition," Geophysical Journal International 215(1), 37-48.

Carcione, J. M., and Helle, H. B. (2004). "The physics and simulation of wave propagation at the ocean bottomwave propagation at ocean bottom," Geophysics 69(3), 825-839.

Chaljub, E., Capdeville, Y., and Vilotte, J.-P. (2003). "Solving elastodynamics in a fluidsolid heterogeneous sphere: a parallel spectral element approximation on non-conforming grids," Journal of Computational Physics 187(2), 457-491.

Cockburn, B., and Shu, C.-W. (2001). "Runge-kutta discontinuous galerkin methods for convection-dominated problems," Journal of Scientific Computing 16(3), 173-261.

De Basabe, J. D., and Sen, M. K. (2014). "A comparison of finite-difference and spectralelement methods for elastic wave propagation in media with a fluid-solid interface," Geophysical Journal International 200(1), 278-298.

de Hoop, A. T., and Van der Hijden, J. H. (1983). "Generation of acoustic waves by an impulsive line source in a fluid/solid configuration with a plane boundary," The Journal of the Acoustical Society of America 74(1), 333-342.

Farnell, G. (1970). "Properties of elastic surface waves," Physical Acoustics 6, 109-166. 
Glorieux, C., Van de Rostyne, K., Gusev, V., Gao, W., Lauriks, W., and Thoen, J. (2002). "Nonlinearity of acoustic waves at solid-liquid interfaces," The Journal of the Acoustical Society of America 111(1), 95-103.

Glorieux, C., Van de Rostyne, K., Nelson, K., Gao, W., Lauriks, W., and Thoen, J. (2001). "On the character of acoustic waves at the interface between hard and soft solids and liquids," The Journal of the Acoustical Society of America 110(3), 1299-1306.

Guo, K., Acosta, S., and Chan, J. (2019). "A weight-adjusted discontinuous galerkin method for wave propagation in coupled elastic-acoustic media," arXiv preprint:1905.09145 .

Hesthaven, J. S., and Warburton, T. (2007). Nodal discontinuous Galerkin methods: algorithms, analysis, and applications (Springer Science \& Business Media).

Komatitsch, D., Barnes, C., and Tromp, J. (2000). "Wave propagation near a fluid-solid interface: A spectral-element approach," Geophysics 65(2), 623-631.

Madec, R., Komatitsch, D., and Diaz, J. (2009). "Energy-conserving local time stepping based on high-order finite elements for seismic wave propagation across a fluid-solid interface," Computer Modeling in Engineering and Sciences (CMES) 14(2), 163.

Padilla, F., de Billy, M., and Quentin, G. (1999). "Theoretical and experimental studies of surface waves on solid-fluid interfaces when the value of the fluid sound velocity is located between the shear and the longitudinal ones in the solid," The Journal of the Acoustical Society of America 106(2), 666-673.

Rauch, D. (1980). "Experimental and theoretical studies of seismic interface waves in coastal waters," in Bottom-Interacting Ocean Acoustics (Springer), pp. 307-327. 
Scholte, J. (1942). "On the stoneley wave equation," Proceedings of the Koninklijke Nederlandse Akademie van Wetenschappen 45(part 1), 20-25.

Stephen, R. (1983). "A comparison of finite difference and reflectivity seismograms for marine models," Geophysical Journal International 72(1), 39-57.

van Vossen, R., Robertsson, J. O., and Chapman, C. H. (2002). "Finite-difference modeling of wave propagation in a fluid-solid configuration," Geophysics 67(2), 618-624.

Warburton, T. (2013). "A low-storage curvilinear discontinuous galerkin method for wave problems," SIAM Journal on Scientific Computing 35(4), A1987-A2012.

Wilcox, L. C., Stadler, G., Burstedde, C., and Ghattas, O. (2010). "A high-order discontinuous galerkin method for wave propagation through coupled elastic-acoustic media," Journal of Computational Physics 229(24), 9373-9396.

Ye, R., de Hoop, M. V., Petrovitch, C. L., Pyrak-Nolte, L. J., and Wilcox, L. C. (2016). "A discontinuous galerkin method with a modified penalty flux for the propagation and scattering of acousto-elastic waves," Geophysical Journal International 205(2), 1267-1289. Zhang, J. (2004). "Wave propagation across fluid - solid interfaces: a grid method approach," Geophysical Journal International 159(1), 240-252. 